\title{
Minimal Discs with Free Boundaries in a Lagrangian Submanifold of $\mathbf{C}^{n}$
}

\author{
Miran ČERne
}

\begin{abstract}
The question when an energy functional stationary disc $p$ with free boundary in a Lagrangian submanifold of $\mathbf{C}^{n}$ is holomorphic or antiholomorphic is considerd. A partial answer is given in terms of its partial indices. It is proved that if all its partial indices are greater or equal to -1 , then the stationary disc $p$ is holomorphic, and if all its partial indices are less or equal to 1 , the disc $p$ is antiholomorphic.
\end{abstract}

1. Introduction. Let $D=\{z \in \mathbf{C} ;|z|<1\}$ and let $\partial D$ be the unit circle in C. For every nonnegative integer $m$ and for every real number $\alpha, 0<\alpha<1$, we denote by $C^{m, \alpha}(D)$ the Banach algebra of $m$-times differentiable complex-valued functions on the closed unit disc $\bar{D}$, whose $m$ derivatives are Hölder continuous with finite Lipschitz norm of the exponent $\alpha$. The subalgebra of functions on $\bar{D}$ which are holomorphic on $D$ we denote by $A^{m, \alpha}(D)$.

Let $M \subseteq \mathbf{C}^{n}$ be a $C^{2}$ manifold and let $p: \bar{D} \rightarrow \mathbf{C}^{n}$ be a disc of class $C^{1, \alpha}$ with boundary in $M$, i.e., $p: \partial D \rightarrow M$. By the energy of the disc $p$ we mean the Dirichlet integral of $p$

$$
E(p):=\frac{1}{2} \int_{D}|\nabla p|^{2} d x d y .
$$

The maps which minimize the energy functional are of a special interest in the Riemannian geometry, namely, any map with boundary in a submanifold $M$ which minimizes energy $E$ in a conjugacy invariant subset $\varnothing \neq G \subseteq \pi_{1}(M)$, also minimizes the area functional

$$
A(p):=\int_{D}\left|\frac{\partial p}{\partial x} \bigwedge \frac{\partial p}{\partial y}\right| d x d y
$$


in $G$-see [Nit] and [Ye2] for more details.

While the existence of the energy functional minimizers with free boundaries in a submanifold $M$ is known in quite general settings, [Ye2], the existence of nontrivial holomorphic discs attached to a certain submanifold of $\mathbf{C}^{n}$ is a much harder problem. It is a well-known and not at all elementary theorem by Gromov, [Gro], that such a disc always exists when the submanifold $M \subseteq \mathbf{C}^{n}$ is a closed Lagrangian submanifold of $\mathbf{C}^{n}$. It is also a well known fact that in this case, i.e., in the case the submanifold $M \subseteq \mathbf{C}^{n}$ is Lagrangian, every holomorphic or antiholomorphic disc attached to $M$ is also an energy functional minimizer with free boundary in $M$. It was Y.-G. Oh's idea, [Oh3], to investigate the reverse implication.

In the present paper we find sufficient conditions on an energy functional stationary disc $p$ with free boundary in a Lagrangian submanifold $M$ of $\mathbf{C}^{n}$ to be holomorphic or antiholomorphic. The main result of the paper is the following theorem.

\section{Theorem 1.1.}

(1) If all partial indices of the pull-back bundle $p^{*}(T M)$ are greater or equal to -1 , then $p$ is a holomorphic disc.

(2) If all partial indices of the pull-back bundle $p^{*}(T M)$ are less or equal to 1 , then $p$ is an antiholomorphic disc.

2. Partial indices. Let $G \ell(n, \mathbf{C})$ be the Lie group of invertible $n \times n$ complex matrices. It is known, see [Vek1], that for every closed path $B: \partial D \rightarrow$ $G \ell(n, \mathbf{C})$ of class $C^{0, \alpha}$ one can find matrix functions

$$
F^{+}: \bar{D} \longrightarrow G \ell(n, \mathbf{C}), \quad F^{-}: \overline{\mathbf{C}} \backslash D \longrightarrow G \ell(n, \mathbf{C})
$$

of class $C^{0, \alpha}$, holomorphic in the interiors of their domains, and $n$ integers $k_{1} \geq$ $k_{2} \geq \ldots \geq k_{n}$ such that

$$
B=F^{+}(\xi) \Lambda(\xi) F^{-}(\xi) \quad(\xi \in \partial D),
$$

where

$$
\Lambda(\xi):=\left(\begin{array}{ccccc}
\xi^{k_{1}} & 0 & \ldots & \ldots & 0 \\
0 & \xi^{k_{2}} & 0 & \ldots & 0 \\
\ldots & \ldots & \ldots & \ldots & \ldots \\
0 & \ldots & \ldots & 0 & \xi^{k_{n}}
\end{array}\right)
$$

The matrix $\Lambda$ is called the characteristic matrix of the path $B$. One can prove that under the condition $k_{1} \geq \ldots \geq k_{n}$, the characteristic matrix $\Lambda$ does not depend on the factorization of the matrix function $B$ of the form above-see 
[Vek1], [Glo1], [Cla-Goh] for more details. The integers $k_{1}, \ldots, k_{n}$ are called the partial indices of the path $B$ and their sum

$$
k:=k_{1}+\cdots+k_{n}
$$

is called the total index of the matrix function $B$.

Let $L$ be a maximal real subspace of $\mathbf{C}^{n}$, i.e., its real dimension is $n$ and $L \oplus i L=\mathbf{C}^{n}$. To every maximal real subspace $L$ one can associate an $\mathbf{R}$-linear map $R_{L}$ on $\mathbf{C}^{n}$, called the reflection about $L$, given by

$$
z=x+i \tilde{x} \longmapsto x-i \tilde{x} \quad(x, \tilde{x} \in L),
$$

where $z=x+i \tilde{x}$ is the unique decomposition of $z$ into the sum of vectors from $L$ and $i L$. The mapping

$$
R_{L}: \mathbf{C}^{n} \longrightarrow \mathbf{C}^{n}
$$

is an $\mathbf{R}$-linear automorphism of $\mathbf{C}^{n}$ that is also $\mathbf{C}$-antilinear, i.e., $R_{L}(i v)=$ $-i R_{L}(v)$ for every $v \in \mathbf{C}^{n}$. The reflection about the maximal real subspace $\mathbf{R}^{n} \subseteq \mathbf{C}^{n}$ will be denoted by $R_{o}$. Note that in the standard notation, $R_{o}$ is just the ordinary conjugation on $\mathbf{C}^{n}$ and that for every matrix $A$ the following identity holds:

$$
\bar{A}=R_{o} A R_{o} .
$$

Let $\langle\cdot, \cdot\rangle$ be the standard Hermitian inner product on $\mathbf{C}^{n}$. By $i L$ we denote the maximal real subspace of vectors of the form $i v, v \in L$, and by $L^{\perp}$ the maximal real subspace of vectors perpendicular to $L$, i.e., a vector $u \in \mathbf{C}^{n}$ is perpendicular to $L$ if and only if $\operatorname{Re}\langle u, v\rangle=0$ for every $v \in L$. The proof of the following simple lemma is omitted; see [Čer1], [Čer2], also [Glo1].

Lemma 2.1. Let $L$ be a maximal real subspace of $\mathbf{C}^{n}$ and let $x_{1}, \ldots, x_{n}$ be any set of vectors spanning $L$. Let $A:=\left[x_{1}, \ldots, x_{n}\right]$ be the matrix whose columns are the given vectors $x_{j}, j=1, \ldots, n$, and let $B_{L}:=A \overline{A^{-1}}$. Then

$$
B_{L}=R_{L} R_{0}
$$

Remark. In the lemma above $n \times n$ matrices $A$ and $B_{L}$ are identified with $\mathbf{C}$-linear automorphisms of $\mathbf{C}^{n}$ in the standard basis.

Corollary 2.1. (a) The matrix $B_{L}$ does not depend on a basis of $L$, (b) $\overline{B_{L}}=B_{L}^{-1}$, (c) $B_{i L}=-B_{L}$, (d) $B_{L^{\perp}}=-B_{L}^{t}$.

The following definition is taken from [Glo1]; see also [For1]. 
Definition 2.1. Let $L=\left\{L_{\xi} ; \xi \in \partial D\right\}$ be a real rank $n$ subbundle of the product bundle $\partial D \times \mathbf{C}^{n}$ of class $C^{0, \alpha}$. If for each $\xi \in \partial D$ the fiber $L_{\xi}$ is a maximal real subspace of $\mathbf{C}^{n}$, the bundle $L$ is called a maximal real bundle of rank $n$ over $\partial D$ and the partial indices of the $G \ell(n, \mathbf{C})$ closed path

$$
B_{L}: \xi \longmapsto R_{L_{\xi}} R_{o}
$$

of class $C^{0, \alpha}$, are called the partial indices of the bundle $L$, and their sum is called the total index of $L$.

Example. Here is a very important example of a maximal real bundle over $\partial D$. Let $M$ be a $C^{1}$ maximal real submanifold of $\mathbf{C}^{n}$ and let $p: \partial D \rightarrow M$ be a $C^{0, \alpha}$ closed curve in $M$. Then the pull-back bundle $p^{*}(T M)$, where $T M$ is the tangent bundle of the submanifold $M$, is a maximal real bundle over $\partial D$ of rank $n$.

Remark. The total index of a closed path $p$ on a maximal real submanifold $M \subseteq \mathbf{C}^{n}$ is also called the Maslov index of $p$.

The special form of the $G \ell(n, \mathbf{C})$ closed path $B_{L}$ implies the following result; see [Glo1], also [Oh1], [Čer1].

Lemma 2.2. The $C^{0, \alpha}$ closed path in $G \ell(n, \mathbf{C})$

$$
B_{L}: \xi \longmapsto R_{L_{\xi}} R_{o}
$$

can be decomposed in the form

$$
B_{L}(\xi)=\Theta(\xi) \Lambda(\xi) \overline{\Theta(\xi)^{-1}} \quad(\xi \in \partial D),
$$

where the map $\Theta: \bar{D} \longrightarrow G \ell(n, \mathbf{C})$ is of class $C^{0, \alpha}$ and holomorphic on $D$, i.e., the $n \times n$ matrix $\Theta$ is in $A^{0, \alpha}(D)^{n \times n}$.

Let $r(\xi), \xi \in \partial D \backslash\{1\}$, denote the principal branch of the square root, i.e., the complex plane is cut along the positive real line and $r(-1)=i$. The characteristic matrix $\Lambda$ can be decomposed further as

$$
\Lambda=\Lambda_{o}^{2}=\Lambda_{o} \overline{\Lambda_{o}^{-1}},
$$

where

$$
\Lambda_{o}(\xi):=\left(\begin{array}{ccccc}
\xi^{k_{1} / 2} & 0 & \ldots & \ldots & 0 \\
0 & \xi^{k_{2} / 2} & 0 & \ldots & 0 \\
\ldots & \ldots & \ldots & \ldots & \ldots \\
0 & \ldots & \ldots & 0 & \xi^{k_{n} / 2}
\end{array}\right)
$$


Here $\xi^{\frac{k}{2}}$ stands for $\xi^{m}$ if $k=2 m$ and for $\xi^{m} r(\xi)$ if $k=2 m+1$. We will refer to $\Lambda_{o}$ as the square root of the characteristic matrix $\Lambda$ and we say that the matrix function

$$
\xi \longmapsto \Theta(\xi) \Lambda_{o}(\xi)
$$

represents a normal form of the bundle $L$.

3. Minimal discs with free boundaries. Let $(r, \vartheta)$ denote the polar coordinates on the unit disc $D$. It is well known, see e.g., [Jäg], [Lew], [Ye2], that any $C^{1, \alpha}$ solution $p$ of the Euler-Lagrange equations for the energy functional $E$ with free boundary in a $C^{2}$ manifold $M$ must satisfy the following conditions:

(a) All component functions of the mapping $p$ are harmonic in $D$, and

(b) $(\partial p / \partial r)(\xi) \perp T_{p(\xi)} M$ for every $\xi \in \partial D$.

Since $p$ maps $\partial D$ into $M$, we also have

$$
\frac{\partial p}{\partial \vartheta}(\xi) \in T_{p(\xi)} M
$$

on $\partial D$. Combining both conditions, we conclude

$$
\frac{\partial p}{\partial r}(\xi) \perp \frac{\partial p}{\partial \vartheta}(\xi)
$$

Since $p$ is harmonic on the unit disk $D$, it can be written in the form

$$
p=f+\bar{g}
$$

for some unique holomorphic vector functions $f$ and $g,(g(0)=0)$, from $\left(A^{1, \alpha}(D)\right)^{n}$. The condition on $p$ to be minimal can be written as

$$
\operatorname{Re}\left\langle\frac{\partial p}{\partial r}(\xi), \frac{\partial p}{\partial \vartheta}(\xi)\right\rangle=0
$$

and so

$$
\operatorname{Re}\left\langle\xi f^{\prime}(\xi)+\overline{\xi g^{\prime}(\xi)}, i \xi f^{\prime}(\xi)-i \overline{\xi g^{\prime}(\xi)}\right\rangle=0 \quad(\xi \in \partial D)
$$

A short calculation shows

$$
\operatorname{Im} \xi^{2}\left\langle f^{\prime}(\xi), \overline{g^{\prime}(\xi)}\right\rangle=0
$$


for every $\xi \in \partial D$. But the function

$$
F(\xi):=\xi^{2}\left\langle f^{\prime}(\xi), \overline{g^{\prime}(\xi)}\right\rangle
$$

extends holomorphically to $D$ and so is a constant function. Since $F(0)=0$, one concludes that

$$
\left\langle f^{\prime}(z), \overline{g^{\prime}(z)}\right\rangle=\sum_{j=1}^{n} f_{j}^{\prime}(z) g_{j}^{\prime}(z)=0
$$

for every $z \in \bar{D}$.

Proposition 3.1. Let $p$ be a complex function $p: \bar{D} \rightarrow \mathbf{C}$ of class $C^{1, \alpha}$. If either of the discs

$$
F, G: \bar{D} \longrightarrow \mathbf{C}^{2}
$$

where

$$
\begin{aligned}
& F(z)=(z, p(z)) \\
& G(z)=(\bar{z}, p(z))
\end{aligned}
$$

is minimal for some $C^{2}$ submanifold $M$ of $\mathbf{C}^{2}$, then the function $p$ is either holomorphic or antiholomorphic.

Proof. Let us assume that the $\operatorname{disc} F(z)=(z, p(z))$ is minimal for some submanifold $M$. Then the corresponding holomorphic discs $f$ and $g$ are of the form

$$
f(z)=(z, a(z)) \quad \text { and } \quad g(z)=(0, b(z))
$$

for some holomorphic functions $a$ and $b$ from $A^{1, \alpha}(D)$. The argument above then implies

$$
a^{\prime}(z) b^{\prime}(z)=0
$$

for every $z \in \bar{D}$. Thus at least one of the functions $a$ and $b$ has to be a constant function. In the case the $\operatorname{disc} G(z)=(\bar{z}, p(z))$ is minimal, the proof is similar.

We would like to use the special normal form of the pull-back bundle to investigate the energy functional minimizers with boundaries in a $C^{2}$ Lagrangian submanifold $M \subseteq \mathbf{C}^{n}$. We recall that a submanifold $M \subseteq \mathbf{C}^{n}$ is called Lagrangian if for each $z \in M$ the tangent space $T_{z} M$ to the submanifold $M$ at the point $z$ is a Lagrangian subspace of $\mathbf{C}^{n}$, i.e.,

$$
\left(T_{z} M\right)^{\perp}=i T_{z} M
$$


Proposition 3.2. Let $L$ be a $C^{0, \alpha}$ maximal real vector bundle of rank $n$ over $\partial D$ such that every fiber $L_{\xi}, \xi \in \partial D$, is a Lagrangian subspace of $\mathbf{C}^{n}$, and let $B_{L}$ be the $C^{0, \alpha}$ closed path in $G \ell(n, \mathbf{C})$ which represents L. Then:

(a) $B_{L}^{*}=B_{L}^{-1}$, i.e., the matrix $B_{L}(\xi)$ is unitary for every $\xi \in \partial D$.

(b) $B_{L}^{t}=B_{L}$, and conversely, if for a maximal real bundle $L$ over $\partial D$ one has $B_{L}^{t}=B_{L}$, then the bundle $L$ is Lagrangian, i.e., each fiber is a Lagrangian subspace of $\mathbf{C}^{n}$.

(c) Let $n_{o}$ denote the number of partial indices of the bundle $L$ that are greater or equal to -1 . Then there are $n \times n_{o}$ and $n \times\left(n-n_{o}\right)$ dimensional matrix functions $X$ and $Y$, respectively, with entries from the space $A^{0, \alpha}(D)$ such that the columns of the matrix function

$$
[X, \bar{Y}] \Lambda_{o}
$$

span the fiber $L_{\xi}$ for each $\xi \in \partial D$. Moreover, $X^{t} Y=0$ on $\bar{D}$. Here $\Lambda_{o}$ is the square root of the corresponding characteristic matrix $\Lambda$.

Remark. In the case $n_{o}$ is $n$ (resp. 0) the matrix $Y$ (resp. $X$ ) does not exist.

Proof. Since the fibers of the bundle $L$ are Lagrangian subspaces of $\mathbf{C}^{n}$, the bundles $i L$ and $L^{\perp}$ are the same. Corollary 2.1 implies $B_{L}^{t}=B_{L}$ and $B_{L}^{*}=B_{L}^{-1}$. This proves (a) and the first part of (b). To prove the reverse implication of part (b), one should observe that if $A$ is any $n \times n$ matrix whose columns span $L_{\xi}$ for some $\xi \in \partial D$, then

$$
A \overline{A^{-1}}=B_{L}(\xi)=B_{L}^{t}(\xi)={\overline{A^{-1}}}^{t} A^{t}=\left(A^{*}\right)^{-1} A^{t} .
$$

So

$$
A^{*} A=A^{t} \bar{A}=\overline{A^{*} A}
$$

and the matrix $A^{*} A$ is a real $n \times n$ matrix. Hence

$$
\operatorname{Re}\left((i A)^{*} A\right)=0
$$

and hence the columns of the matrix $i A$ are perpendicular to the columns of the matrix $A$. Part (b) is proved.

To prove part (c) we use the factorization of the matrix function $B_{L}$,

$$
B_{L}=\Phi \Lambda \overline{\Phi^{-1}},
$$

for some map $\Phi: \bar{D} \rightarrow G \ell(n, \mathbf{C})$ from $A^{0, \alpha}(D)^{n \times n}$. We define $\Psi:=\left(\Phi^{-1}\right)^{*}$ and by part (b) we also have

$$
B_{L}=\Psi \Lambda \overline{\Psi^{-1}} .
$$


Let

$$
\Phi=\left[\Phi_{1}, \Phi_{2}\right] \quad \text { and } \quad \Psi=\left[\Psi_{1}, \Psi_{2}\right]
$$

be the block notation of the matrix functions $\Phi$ and $\Psi$ such that the matrices $\Phi_{1}$ and $\Psi_{1}$ have dimensions $n \times n_{o}$. Since

$$
\Phi^{t} \bar{\Psi}=\Phi^{t}\left(\Phi^{-1}\right)^{t}=I_{n},
$$

we get

$$
\left[\begin{array}{l}
\Phi_{1}^{t} \\
\Phi_{2}^{t}
\end{array}\right]\left[\overline{\Psi_{1}}, \overline{\Psi_{2}}\right]=I_{n}
$$

and so

$$
\Phi_{1}^{t} \overline{\Psi_{2}}=0 .
$$

This means that the columns of the matrix $\Phi_{1}$ are orthogonal to the columns of the matrix $\Psi_{2}$, and thus the matrix

$$
\left[\Phi_{1}, \Psi_{2}\right]
$$

is invertible. We set $X:=\Phi_{1}$ and $Y:=\overline{\Psi_{2}}$ and the proof is finished.

The proof of part (c) of the proposition above also implies the following result.

Corollary 3.2. Let $L$ be a Lagrangian vector bundle over $\partial D$ of class $C^{0, \alpha}$, let $B_{L}$ be the corresponding $C^{0, \alpha}$ closed path in $G \ell(n, \mathbf{C})$ and let $\Lambda$ be its characteristic matrix. Then the characteristic matrix of the $G \ell(n, \mathbf{C})$ closed path

$$
\xi \longmapsto B_{L}(\bar{\xi})
$$

is $\bar{\Lambda}$.

Remark. Statement (c) of the proposition above does not hold for an arbitrary maximal real vector bundle over the $\partial D$. As a counterexample, one can take the bundle $L$ whose matrix $B_{L}$ is given by

$$
\left(\begin{array}{cc}
-\xi^{2} & 1 \\
0 & \bar{\xi}^{2}
\end{array}\right)
$$


It is easy to check that the partial indices of $B_{L}$ are 2 and -2 and that there are no nontrivial holomorphic functions $a, b \in A^{0, \alpha}(D)$ that satisfy the equation

$$
\left(\begin{array}{cc}
-\xi^{2} & 1 \\
0 & \bar{\xi}^{2}
\end{array}\right)\left(\begin{array}{l}
a(\xi) \\
b(\xi)
\end{array}\right)=\bar{\xi}^{2}\left(\begin{array}{l}
\overline{a(\xi)} \\
\overline{b(\xi)}
\end{array}\right) \quad(\xi \in \partial D) .
$$

Henceforth $M$ will denote a $C^{2}$ Lagrangian submanifold in $\mathbf{C}^{n}$ and $p: \bar{D} \rightarrow$ $\mathbf{C}^{n}$ will be an energy functional stationary disc of class $C^{1, \alpha}$ with boundary in $M$, i.e., the first variation of the energy functional $E$ at $p$ is 0 . We recall that the pair $(r, \vartheta)$ represents the polar coordinates on $D$. Then one has

$$
\xi \frac{\partial p}{\partial z}(\xi)=\frac{1}{2}\left(\frac{\partial p}{\partial r}(\xi)-i \frac{\partial p}{\partial \vartheta}(\xi)\right)
$$

and

$$
\bar{\xi} \frac{\bar{\partial} p}{\partial \bar{z}}(\xi)=\frac{1}{2}\left(\frac{\partial p}{\partial r}(\xi)+i \frac{\partial p}{\partial \vartheta}(\xi)\right)
$$

for every $\xi \in \partial D$. The disc $p$ is attached to the manifold $M$; hence, the vector $(\partial p / \partial \vartheta)(\xi)$ is tangent to $M$ at $p(\xi)$ for each $\xi \in \partial D$. Since $p$ is also minimal, the vector $(\partial p / \partial r)(\xi)$ is perpendicular to $M$ at $p(\xi)$ for every $\xi \in \partial D$.

Let $A_{o}$ denote the matrix constructed in part (c) of Proposition 3.2. Since $M$ is a Lagrangian submanifold of $\mathbf{C}^{n}$, we get

$$
\operatorname{Re}\left(i A_{o}^{*} \frac{\partial p}{\partial \vartheta}(\xi)\right)=0 \quad(\xi \in \partial D)
$$

and since the first variation of the energy functional $E$ at $p$ vanishes, we have

$$
\operatorname{Re}\left(A_{o}^{*} \frac{\partial p}{\partial r}(\xi)\right)=0 \quad(\xi \in \partial D) .
$$

Thus on $\partial D$ the following identities hold:

$$
\operatorname{Re}\left(\bar{\xi} A_{o}^{*}(\xi) \frac{\partial p}{\partial \bar{z}}(\xi)\right)=0 \quad \text { and } \quad \operatorname{Re}\left(\xi A_{o}^{*}(\xi) \frac{\partial p}{\partial z}(\xi)\right)=0 .
$$

We know that the disc $p$ can be decomposed into a sum $p=f+\bar{g}$ for some unique vector functions $f$ and $g,(g(0)=0)$, from $A^{1, \alpha}(D)^{n}$. Therefore, we have

$$
\operatorname{Re}\left(\xi A_{o}^{t}(\xi) g^{\prime}(\xi)\right)=0 \quad \text { and } \quad \operatorname{Re}\left(\xi A_{o}^{*}(\xi) f^{\prime}(\xi)\right)=0
$$


for every $\xi \in \partial D \backslash\{1\}$. Since also $A_{o}=[X, \bar{Y}] \Lambda_{o}$, where the matrices $X$ and $Y$ have holomorphic extensions into $D$ and are "orthogonal" to each other, i.e., $Y^{t} X=0$ on $\bar{D}$, we first conclude that

$$
X^{t} g^{\prime}=0 \quad \text { and } \quad Y^{t} f^{\prime}=0,
$$

and finally

$$
f^{\prime}=X h \quad \text { and } \quad g^{\prime}=Y k
$$

for some vector functions $h \in\left(A^{0, \alpha}(D)\right)^{n_{o}}$ and $k \in\left(A^{0, \alpha}(D)\right)^{n-n_{o}}$. For these results we used only one part of each equation in (1). The rest, together with the equalities above, implies

$$
\operatorname{Re}\left(\xi \overline{\Lambda_{o}^{+}} X^{*} X h\right)=0 \quad \text { and } \quad \operatorname{Re}\left(\xi \overline{\Lambda_{o}^{-}} Y^{*} Y k\right)=0,
$$

where $\Lambda_{o}^{+}$and $\Lambda_{o}^{-}$are the $n_{o} \times n_{o}$ and the $\left(n-n_{o}\right) \times\left(n-n_{o}\right)$ dimensional matrices, respectively, such that

$$
\Lambda_{o}=\left(\begin{array}{cc}
\Lambda_{o}^{+} & 0 \\
0 & \overline{\Lambda_{o}^{-}}
\end{array}\right) .
$$

The manifold $M$ is Lagrangian, thus

$$
\operatorname{Re}\left(-i A_{o}^{*} A_{o}\right)=0
$$

on $\partial D$, and the matrices

$$
\overline{\Lambda_{o}^{+}} X^{*} X \Lambda_{o}^{+} \quad \text { and } \quad \overline{\Lambda_{o}^{-}} Y^{*} Y \Lambda_{o}^{-}
$$

are real and invertible. Hence

$$
\operatorname{Re}\left(\xi \overline{\Lambda_{o}^{+}(\xi)} h(\xi)\right)=0 \quad \text { and } \quad \operatorname{Re}\left(\xi \overline{\Lambda_{o}^{-}(\xi)} k(\xi)\right)=0
$$

for every $\xi \in \partial D$. This proves the following theorem.

Theorem 3.1. Let $M$ be a $C^{2}$ Lagrangian submanifold of $\mathbf{C}^{n}$ and let $p=$ $f+\bar{g}, f, g \in\left(A^{1, \alpha}(D)\right)^{n}$, be an energy functional stationary disc of class $C^{1, \alpha}$ with boundary in $M$. Let $n_{o}$ be the number of partial indices of the path $p(\partial D) \subseteq$ $M$ which are greater or equal to -1 , and let $X$ and $Y$ be the $n \times n_{o}$ and $n \times\left(n-n_{o}\right)$ dimensional holomorphic matrices given by part (c) of Proposition 3.2. Then there exist vector functions $h \in\left(A^{0, \alpha}(D)\right)^{n_{o}}$ and $k \in\left(A^{0, \alpha}(D)\right)^{n-n_{o}}$ such that

$$
\operatorname{Re}\left(\xi \overline{\Lambda_{o}^{+}(\xi)} h(\xi)\right)=0 \text { and } \operatorname{Re}\left(\xi \overline{\Lambda_{o}^{-}(\xi)} k(\xi)\right)=0 \quad(\xi \in \partial D)
$$


and

$$
f^{\prime}=X h \quad \text { and } \quad g^{\prime}=Y k .
$$

Remark. In the case $n_{o}$ equals $n$ (resp. 0), one part of the conclusion above is empty, namely, the matrix $Y$ (resp. $X$ ) does not exist.

As a simple consequence, one has the following result:

Corollary 3.3. (Hypothesis as above.)

(a) If all partial indices of the pull-back bundle $p^{*}(T M)$ are greater or equal to -1 , then $p$ is a holomorphic disc.

(b) If all partial indices of the pull-back bundle $p^{*}(T M)$ are less or equal to 1 , then $p$ is an antiholomorphic disc.

4. Acknowledgements. I would like to thank my thesis advisor Professor Franc Forstnerič for his help and constant encouragement. I am indebted to Professor Yong-Geun Oh for his seminar talk and stimulating discussions.

This work was supported in part by a grant from the Ministry of Science and Technology of the Republic of Slovenia.

\section{REFERENCES}

[Cla-Goh] K. Clancey and I. Gohberg, Factorization of matrix functions and singular integral operators. Birkhäuser Verlag, Basel-Boston-Stuttgart 1981.

[Čer1] M. Černe, Ph. D. Thesis. Univ. of Wisconsin, Madison, 1994.

[Čer2] M. Černe, Analytic discs attached to a generating CR-manifold, Ark. Mat. (to appear).

[For1] F. Forstnerič, Analytic discs with boundaries in a maximal real submanifolds of $\mathbf{C}^{2}$, Ann. Inst. Fourier 37 (1987), 1-44.

[For2] F. Forstnerič, Polynomial hulls of sets fibered over the circle, Indiana Univ. Math. J. 37 (1988), 869-889.

[Glo1] J. GLobevnik, Perturbation by analytic discs along maximal real submanifold of $\mathbf{C}^{n}$, Math. Z. 217 (1994), 287-316.

[Glo2] J. GLobevnik, Perturbing analytic discs attached to maximal real submanifolds of $\mathbf{C}^{n}$ (Preprint).

[Gro] M. Gromov, Pseudo-holomorphic curves in symplectic manifolds, Invent. Math. 81 (1985), 307-347.

[Jäg] W. JäGER, Behavior of minimal surfaces with free boundaries, Comm. Pure Appl. Math. 23 (1970), 803-818.

[Lew] H. Lewy, On the boundary behavior of minimal surfaces Proc. Natl. Acad. Sci. 37 (1951), 103-110.

[Nit] J. C. C. Nitsche, Minimal surfaces with partially free boundary. Least area property and Hölder continuity for boundaries satisfying a chordarc condition., Arch. Ration. Mech. Anal. 39 (1970), 131-145.

[Oh1] Y.-G. Он, The Fredholm-regularity and realization of the RiemannHilbert problem and application to the perturbation theory of analytic discs (Preprint). 
[Oh2] Y.-G. Он, Fredholm theory of holomorphic discs with Lagrangian or totally real boundary conditions under the perturbation of boundary conditions (Preprint).

[Oh3] Y.-G. Он. Seminar talk.

[Vek1] N. P. Vekua, Systems of singular integral equations. Nordhoff, Groningen 1967.

[Ye1] R. Ye, Gromov's compactness theorem for pseudo-holomorphic curves (Preprint).

[Ye2] R. YE., On the existence of area-minimizing surfaces with free boundary, Math. Z. 206 (1991), 321-331.

Department of Mathematics and Mechanics

University of Ljubljana

Jadranska 19, 61111 Ljubljana

Slovenia

Received: July 6th, 1994. 